Sädhanā, Vol. 18, Part 5, September 1993, pp. 731-747. (C) Printed in India.

\title{
Stability theory of differential/algebraic models of power systems
}

\section{J HILL, I A HISKENS and I M Y MAREELS}

Department of Electrical and Computer Engineering, University of Newcastle, Australia

\begin{abstract}
Lyapunov stability results are given for differential/algebraic inodels of power systems which include the effect of generator damping and nonlinear loads. The global dynamical structure of such a system is studied in terms of multivalued energy functions defined on so-called 'voltage causal regions' where voltage behaviour is predicted from angle behaviour. These regions are separated by 'impasse surfaces' related to singularity in the load flow equations.
\end{abstract}

Keywords. Power systems; stability; Lyapunov methods; nonlinear systems.

\section{Introduction}

Direct methods of transient stability assessment for power systems rely on simplified nonlinear equation models. Older models assumed impedance loads and used network reduction to derive a model as a set of coupled (swing) differential equations (Pai 1981). Bergen \& Hill (1981) suggested using models where the loads and network structure were preserved. This approach leads naturally to models which are of diffcrential/algebraic type. More recently such models have been used as a basis for voltage stability analysis (Kwatny et al 1986; Venkatasubramanian et al 1991). Thus the theoretical basis for direct methods using structure-preserving models is dependent on stability theory for differential/algebraic equations (Chiang \& Fekih-Ahmed 1992). This paper provides further results in that direction.

Hill \& Mareels (1990) have given some basic results on Lyapunov stability of differential/algebraic systems and used these to justify use of an energy function for undamped power systems. Hiskens \& Hill (1989) have explored more practical aspects of using this energy function; this work identifies several theoretical extensions which should be marie. Of these, the two considered here are as follows. First., the theory is easily extended to allow for generator damping. Second, an improved decomposition of the state-space is presented; so-called voltage causal regions are defined as open sets which are separated by 'impasse surfaces' of algebraic singularity and within which ordinary differential equation theory can be used. This helps to formulate practical algorithms for finding the region of transient stability. Further, there are useful tools for analysis of short-term voltage collapse. 


\section{Differential/algebraic power system model}

\subsection{Model development}

The classical machine model is used in the development of the system model. Therefore the synchronous machines are represented by a constant voltage $\left|E_{i}\right|$ in series with transient reactance. This assumption corresponds to ignoring flux decay and having an exciter which is too slow to act in the transient period.

Consider now a network consisting of $n_{0}$ buses connected by transmission lines. At $m$ of these buses there are generators. The buses which have load but no generation are labelled $i=1, \ldots, n_{0}-m$. The network is augmented with $m$ fictitious buses representing the generator internal buses, in accordance with the classical machine model. They are labelled $i+m$ where $i$ is the bus number of the corresponding generator bus. The total number of buses in the augmented system is therefore $n_{0}+m:=n$.

The network is assumed lossless, so all lines (including those corresponding to the machine transient reactances) are modelled as series reactances. The bus admittance matrix $\mathrm{Y}$ is therefore purely imaginary, with elements $Y_{i j}=j B_{i j}$.

Let the complex voltage at the ith bus be the (time varying) phasor $V_{i}=\left|V_{i}\right| \angle \delta_{i}$ where $\delta_{i}$ is the bus phase angle with respect to a synchronously rotating reference frame. Define $|\mathrm{V}|=\left[\left|V_{1}\right|, \ldots,\left|V_{n_{0}}\right|\right]^{i}$, where $t$ denotes matrix transpose. The bus frequency deviation is given by $\omega_{i}=\dot{\delta}_{i}$.

Using machine reference angles, we take the $n$th bus as the reference. We use the internodal angles $\alpha_{i}:=\delta_{i}-\delta_{n}$. Define $\alpha=\left[\alpha_{1}, \ldots, \alpha_{n-1}\right]^{t}$ and $\omega_{g}=\left[\omega_{n_{0}}+1, \ldots, \omega_{n}\right]^{?}$.

Let $P_{b i}$ and $Q_{b i}$ denote the total real and reactive power leaving the $i$ th bus via transmission lines. Then

$$
\begin{aligned}
& P_{b i}(\alpha,|\mathbf{V}|)=\sum_{j=1}^{n}\left|V_{i}\right|\left|V_{j}\right| B_{i j} \sin \left(\alpha_{i}-\alpha_{j}\right) \\
& Q_{b i}(\alpha,|\mathbf{V}|)=-\sum_{j=1}^{n}\left|V_{i}\right|\left|V_{j}\right| B_{i j} \cos \left(\alpha_{i}-\alpha_{j}\right) .
\end{aligned}
$$

In these equations, we assume the substitution $\left|V_{i}\right|=\left|E_{i-n 0}\right|, i=n_{0}+1, \ldots, n$ has been made. Also we take $\alpha_{n}:=0$.

Now consider the modelling of loads. Denote the real and reactive power demand at the $i$ th bus by $P_{d i}$ and $Q_{d i}$ respectively. In general these powers are functions of voltage and frequency. For the stability theory to be developed, the loads must be restricted to satisfy

$$
\begin{aligned}
& P_{d i}=P_{d i}^{0}, \\
& Q_{d i}=Q_{d i}\left(\left|V_{i}\right|\right), \quad i=1, \ldots, n_{0} .
\end{aligned}
$$

There are unresolved difficulties in allowing voltage dependent real loads. However, this restriction will be relaxed when considering the state space decomposition.

The last component of the model to be considered is the generator dynamics, given by the swing equations

$$
M_{j}\left(\mathrm{~d} \omega_{j+t_{0}} / \mathrm{d} t\right)+D_{j} \omega_{j+n_{0}}+P_{b, j+n_{0}}(\boldsymbol{\alpha},|\mathbf{V}|)=P_{M j}^{0} ; j=1, \ldots, m,
$$


where $M_{j}$ are the inertia constants, $D_{j}$ the generator damping constants, and $P_{M j}^{0}$ the mechanical input powers. We assume that $D_{i} \neq 0$ for at least one $i=1, \ldots, m$. The usual assumptions of constant mechanical power, and the network being in a sinusoidal steady state are made.

Combining the power balance and swing equations gives the total system representation

$$
\begin{aligned}
M_{i-n_{0}} \dot{\omega}_{i}+D_{i-n_{0}} \omega_{i}+P_{b i}(\boldsymbol{\alpha},|\mathbf{V}|) & =P_{M, i-n_{0}}^{0}, & & i=n_{0}+1, \ldots, n, \\
P_{b i}(\alpha,|\mathbf{V}|) & =-P_{d i}^{0}, & & i=1, \ldots, n_{0}, \\
Q_{b i}(\alpha,|\mathbf{V}|) & =-Q_{d i}\left(\left|V_{i}\right|\right), & & i=1, \ldots, n_{0} .
\end{aligned}
$$

Define $\tilde{\mathbf{P}}_{b}^{t}=\left[P_{b 1}, \ldots, P_{b, n-1}\right]=\left[\mathbf{P}_{l}^{t} \mathbf{P}_{g}^{t}\right]$ where $\mathbf{P}_{l}, \mathbf{P}_{g}$ are $n_{0},(m-1)$ vectors referring to loads, generators respectively; $\tilde{\mathbf{P}}^{0 t}=\left[-\mathbf{P}_{d}^{0 t} \tilde{\mathbf{P}}_{M}^{0 t}\right]$ where $\widetilde{\mathbf{P}}_{M}^{0}=\left[P_{M 1}^{0}, \ldots, P_{M, m-1}^{0}\right]^{t}$; $\mathbf{Q}_{b}=\left[Q_{b 1}, \ldots, Q_{b n o}\right]^{t} ;$ and $\mathbf{Q}=\left[-Q_{d 1}, \ldots,-Q_{d n o}\right]^{t}$. Also set $P_{n}:=P_{M m}^{0}$.

From (1a)

$$
\sum_{i=1}^{n}\left(P_{b i}-P_{i}^{0}\right)=-\sum_{i=1}^{n} P_{i}^{0}:=-P_{T}
$$

It is convenient for this excess bus power to be distributed across generator powers in proportion to damping. Define

$$
P_{M, j}=P_{M, j}^{0}-\left(P_{T} / D_{T}\right) D_{j}
$$

where $D_{T}:=\sum_{i=1}^{m} D_{i}(\neq 0$ by assumption $)$.

Define the modified real power vector $\tilde{\mathbf{P}}^{t}=\left[-\mathbf{P}_{d}^{0 t} \tilde{\mathbf{P}}_{M}^{t}\right]$. Then from (4b), (5), (6)

So

$$
\sum_{i=n_{0}+1}^{n}\left(P_{b i}-P_{i}\right)=0
$$

$$
P_{b n}-P_{n}=-\mathbf{I}_{n-1}^{t}\left(\mathbf{P}_{g}-\tilde{\mathbf{P}}_{M}\right)
$$

where $\mathbf{I}_{n-1}$ is the $(n-1)$ vector with unity entries. Define $\mathbf{T}_{g}=\left[\mathbf{I}_{n-1} ;-\mathbf{I}_{n-1}\right]$ where $I_{n-1}$ is the $(n-1)$ identity matrix. Then (4) can be rewritten

$$
\begin{array}{r}
\mathbf{M}_{g} \dot{\boldsymbol{\omega}}_{g}+\mathbf{D}_{\theta} \omega_{g}+\mathbf{T}_{g}^{t}\left(\mathbf{P}_{g}(\alpha,|\mathbf{V}|)-\tilde{\mathbf{P}}_{M}\right)=\mathbf{0} \\
\mathbf{P}_{1}(\boldsymbol{\alpha},|\mathbf{V}|)+\mathbf{P}_{d}=\mathbf{0} \\
\mathbf{Q}_{b}(\alpha,|\mathbf{V}|)+\mathbf{Q}_{d}(|\mathbf{V}|)=\mathbf{0}
\end{array}
$$

where $\mathbf{M}_{g}, \mathbf{D}_{g}$ are diagonal matrices of inertia, damping constants. Note that use of $\tilde{\mathbf{P}}_{M}$ requires a reference shift for $\dot{\omega}_{g}$ so that (4a) remains valid. Partition $\alpha$ as $\alpha^{t}=\left[\alpha_{l}^{t} \alpha_{g}^{t}\right]$ so the loads can be identified.

Also define

$$
\begin{aligned}
\tilde{\mathbf{f}}_{g}\left(\boldsymbol{\alpha}_{g}, \boldsymbol{\alpha}_{l},|\mathbf{V}|\right): & =\mathbf{P}_{\theta}\left(\boldsymbol{\alpha}_{g}, \boldsymbol{\alpha}_{l},|\mathbf{V}|\right)-\tilde{\mathbf{P}}_{M}, \\
\mathbf{f}_{l}\left(\boldsymbol{\alpha}_{g}, \boldsymbol{\alpha}_{l},|\mathbf{V}|\right): & =\mathbf{P}_{l}\left(\boldsymbol{\alpha}_{\theta}, \boldsymbol{\alpha}_{l},|\mathbf{V}|\right)+\mathbf{P}_{d} \\
\mathbf{g}\left(\boldsymbol{\alpha}_{g}, \boldsymbol{\alpha}_{l},|\mathbf{V}|\right): & =[|\mathbf{V}|]^{-1}\left(\mathbf{Q}_{b}\left(\boldsymbol{\alpha}_{g}, \boldsymbol{\alpha}_{l},|\mathbf{V}|\right)+\mathbf{Q}_{d}(|\mathbf{V}|)\right),
\end{aligned}
$$


where [a] denotes $\left[\right.$ diag $\left.\left\{a_{i}\right\}\right]$ for vector a. Then (7) can be written

$$
\begin{aligned}
\dot{\omega}_{g} & =-\mathbf{M}_{g}^{-1} \mathbf{D}_{g} \omega_{g}-\mathbf{M}_{g}^{-1} \mathbf{T}_{g}^{\prime} \tilde{\mathbf{f}}_{g}\left(\alpha_{g}, \boldsymbol{\alpha}_{l},|\mathbf{V}|\right), \\
\dot{\alpha}_{g} & =\mathbf{T}_{g} \omega_{g}, \\
\mathbf{0} & =\mathbf{f}_{l}\left(\boldsymbol{\alpha}_{g}, x_{l},|\mathbf{V}|\right), \\
\mathbf{0} & =\mathbf{g}\left(\boldsymbol{\alpha}_{g}, \boldsymbol{\alpha}_{l}|\mathbf{V}|\right) .
\end{aligned}
$$

Equations (9), (10) describe the model on which all further results are based. We note it consists of a set of differential-algebraic (DA) equations. The system variables are clearly

$$
\omega_{g} \in \mathbb{R}^{m}, \alpha_{g} \in \mathbb{R}^{m-1}, \alpha_{l} \in \mathbb{R}^{n_{0}} \text { and }|V| \in \mathbb{R}_{+}^{n_{0}} .
$$

It is easy to check that the bus power transformation (and associated frequency reference shift) implies the equilibrium points are given by $\omega_{\theta}=0$ and

$$
\begin{aligned}
& \tilde{\mathbf{f}}_{g}\left(\alpha_{g}, \alpha_{l},|\mathbf{V}|\right)=0, \\
& \mathbf{f}_{l}\left(\alpha_{g}, \alpha_{l},|\mathbf{V}|\right)=0, \\
& g\left(\alpha_{y}, \alpha_{l},|\mathbf{V}|\right)=0 .
\end{aligned}
$$

\subsection{Local ODE representation}

Here it is shown that the model is locally equivalent to a set of ordinary differential equations (ODE) for almost all operating states. The load bus variables $\boldsymbol{\alpha}_{i},|\mathbf{V}|$ are related to the generator angles $\alpha_{\beta}$ by the algebraic equations (10). In fact, (10) defines an $(m-1)$-manifold on which $\alpha_{g}$ can flow. Define the Jacobian

$$
J_{u}=\left[\begin{array}{ll}
\partial \mathbf{f}_{l} / \partial \boldsymbol{\alpha}_{l} & \partial \mathbf{f}_{l} / \hat{\sigma}|\mathbf{V}| \\
\partial \mathbf{g} / \partial \boldsymbol{\alpha}_{l} & \partial \mathbf{g} / \hat{c}|\mathbf{V}|
\end{array}\right]
$$

Then, by the implicit function theorem (Flerning 1977), if det $J_{11} \neq 0$, locally the load bus variables can be written explicitly in terms of the generator angles as

$$
\alpha_{1}=\boldsymbol{\Phi}\left(\alpha_{g}\right),|\mathbf{V}|=\Psi\left(\alpha_{g}\right)
$$

An equivalent differential equation form can therefore be obtained locally by substituting (13) into (9a). Setting

$$
\mathbf{P}_{g}^{*}\left(\alpha_{g}\right):=\mathbf{P}_{g}\left(\alpha_{g}: \Phi\left(\alpha_{g}\right), \Psi\left(\alpha_{g}\right)\right)
$$

gives the model

$$
\begin{aligned}
& \dot{\omega}_{g}=-\mathbf{M}_{g}^{-1} \mathbf{D}_{g} \boldsymbol{\omega}_{g}-\mathbf{M}_{g}^{-1} \mathbf{T}_{g}^{t}\left(\mathbf{P}_{g}^{*}\left(\alpha_{g}\right)-\tilde{\mathbf{P}}_{M}\right), \\
& \dot{\alpha}_{g}=\mathbf{T}_{g} \omega_{g} .
\end{aligned}
$$

Equations (14) define ordinary differential equations which are locally equivalent to the DA system.

This idea of local solvability will be extended later to solvability over disjoint regions. 


\section{Stability theory of differential/algebraic systems}

This section develops a useful result on the stability of equilibria in general DA systems. The general topic of Lyapunov stability for such systems has been studied in Hill \& Mareels (1990). The result required here is a LaSalle invariance version of an asymptotic stability criterion. This is easily developed using ideas given in Hill \& Mareels (1990).

We consider $\mathrm{DA}$ in the general form

$$
\begin{aligned}
& \dot{\mathbf{x}}=\mathbf{f}(\mathbf{x}, \mathbf{y}), \\
& \mathbf{0}=\mathbf{g}(\mathbf{x}, \mathbf{y}),
\end{aligned}
$$

with some compatible initial conditions, $\left(\mathbf{x}_{0}, \mathbf{y}_{0}\right)$, i.e. $\mathbf{0}=\mathbf{g}\left(\mathbf{x}_{0}, \mathbf{y}_{0}\right)$ where $\mathbf{f}: \mathbb{R}^{n} \times \mathbb{R}^{m} \rightarrow \mathbb{R}^{n}, \mathbf{g}: \mathbb{R}^{n} \times \mathbb{R}^{m} \rightarrow \mathbb{R}^{m}$.

We assume throughout:

A1. f and $\mathbf{g}$ are twice continuously differentiable in some open connected set, $\Omega \subset \mathbb{R}^{n} \times \mathbb{R}^{m}$, i.e. $\mathbf{f}, \mathbf{g} \in C^{2}(\Omega)$.

A2. The Jacobian of $g$ with respect by $\mathbf{y}$ has constant full rank on $\Omega$, i.e.

$$
\operatorname{rank}\left(D_{2} \mathbf{g}(\mathbf{x}, \mathbf{y})\right)=m, \quad \forall(\mathbf{x}, \mathbf{y}) \in \Omega .
$$

We use the following notations:

$\mathbf{x}\left(t, \mathbf{x}_{0}, \mathbf{y}_{0}\right), \mathbf{y}\left(t, \mathbf{x}_{0}, \mathbf{y}_{0}\right)$ are solutions of (15) as functions of time and initial conditions

$$
\begin{aligned}
& B_{c}=\left\{(\mathbf{x}, \mathbf{y}) \in \mathbb{R}^{n} \times \mathbb{R}^{m}:\|(\mathbf{x}, \mathbf{y})\|<\varepsilon\right\}, \\
& G=\left\{(\mathbf{x}, \mathbf{y}) \in \mathbb{R}^{n} \times \mathbb{R}^{m}: \mathbf{g}(\mathbf{x}, \mathbf{y})=0\right\}, \\
& \Omega_{G}=\Omega \cap G, \\
& \bar{\Omega}=\text { closure of } \Omega \text { in } \mathbb{R}^{n} \times \mathbb{R}^{m}, \\
& K=\left\{a: \mathbb{R}_{+} \rightarrow \mathbb{R}_{+} \text {continuous, strictly increasing, } a(0)=0\right\}, \\
& \dot{V}_{(n)}=\text { derivative of the function } V \text { with respect to time along the solution } \\
& \text { of the system with equations }(n) .
\end{aligned}
$$

We now consider stability properties of equilibria of the general DA system (15). First, note that a local ODE description - exemplified by (14) - can be given.

It follows from the implicit function theorem and assumption $\mathrm{A} 2$ that given $(\overline{\mathbf{x}}, \overline{\mathbf{y}}) \in \Omega_{G}$ there is some neighbourhood $U \subset \mathbb{R}^{n}$ of $\overline{\mathbf{x}}$ and a unique twice differentiable function $\mathbf{u}: \mathbb{R}^{n} \rightarrow \mathbb{R}^{m}, \mathbf{u} \in C^{2}(U)$ such that

$$
\mathbf{0}=\mathbf{g}(\mathbf{x}, \mathbf{u}(\mathbf{x})) \forall \mathbf{x} \in U \text { and }(U \times \mathbf{u}(U))_{G} \subset \Omega_{G},
$$

with Jacobian

Let

$$
(D \mathbf{u})(\mathbf{x})=-\left(D_{2} \mathbf{g}\right)^{-1}(\mathbf{x}, \mathbf{u}(\mathbf{x})) \cdot\left(D_{1} \mathbf{g}\right)(\mathbf{x}, \mathbf{u}(\mathbf{x})), \forall \mathbf{x} \in U .
$$

$$
\Lambda:=U \times \mathbf{u}(U) \text { and } \Lambda_{G}:=(U \times \mathbf{u}(U))_{G}=(U \times \mathbf{u}(U)) \cap G
$$

Lemma 1. In the neighbourhood $\Lambda_{G}(\bar{x}) \subset \Omega_{G}$, the system (15) reduces to

$$
\dot{x}=\mathbf{f}(x, u(x)) \text {. }
$$


We assurne that the system (15) has a unique (isolated) equilibrium in $\Omega$, which we regard to be the origin, without loss of generality.

A3. In $\Omega, f(x, y)=0$ and $g(x, y)=0$, iff $(x, y)=(0,0)$.

Remark. In order to satisfy assumption A3 it may be necessary to shrink the set $\Omega$ of assumptions $\mathrm{A} 1$ and $\mathrm{A} 2$ to a smaller subset.

When discussing stability in the DA system context we only consider stability with respect to perturbations which satisfy the algebraic constraints. (When using the reduced system representation (16), this feature has been accounted for.) However it should be noted that often the algebraic equations of the DA system are a model of some (perhaps unknown) underlying dynamic process. Stability in the DA sense does not imply stability of the system obtained by including the underlying dynamics.

We now present the formal definitions of stability of the trivial solution $(\mathbf{x}(\mathbf{t}, \mathbf{0}, \mathbf{0})$, $y(t, 0,0)) \equiv(0,0)$ of the DA system (15).

\section{DEFINITION 1}

The trivial solution of (15) is called stable if given $\varepsilon>0$, there exists a $\delta>0$ such that for all $\left(\mathbf{x}_{0}, \mathbf{y}_{0}\right) \in \Omega_{G} \cap B_{\delta}$ then $\left(\mathbf{x}\left(t, \mathbf{x}_{0}, \mathbf{y}_{0}\right), \mathbf{y}\left(t, \mathbf{x}_{0}, \mathbf{y}_{0}\right)\right) \in \Omega_{G} \cap B_{s}, \forall t \in \mathbb{R}_{+}$.

\section{DEFINITION 2}

The trivial solution of $(15)$ is called asymptoticaliy stable if it is stable and there exists $\eta>0$ such that for all $\left(\mathbf{x}_{0}, \mathbf{y}_{0}\right) \in \Omega_{G} \cap B_{\eta}$ then

$$
\lim _{t \rightarrow \infty}\left\|\left(\mathbf{x}\left(t, \mathbf{x}_{0}, \mathbf{y}_{0}\right), \mathbf{y}\left(t, \mathbf{x}_{0}, \mathbf{y}_{0}\right)\right)\right\|=(\mathbf{0}, \mathbf{0}) \text {. }
$$

It is straightforward to derive versions of the basic Lyapunov stability arguments for DA systems. Some basic results are given in Hill \& Mareels (1990). In the latter power system analysis we need a LaSalle invariance type result.

Theorem 1. Suppose there exists a $C^{\mathbf{1}}(\Omega)$ function $V: \Omega \rightarrow \mathbb{R}_{+}$such that $V$ is positive definite and has negative semi-definite derivative on $\Omega_{G}$, i.e.

$$
\begin{aligned}
& V(\mathbf{x}, \mathbf{y}) \geqslant a(\|(\mathbf{x}, \mathbf{y})\|), \\
& \dot{V}_{(15)} \leqslant 0,
\end{aligned}
$$

on $\Omega_{G}$ for some $a \in K$. Let

$$
S=\left\{(\mathbf{x}, \mathbf{y}) \in \Omega_{G} \cap \Lambda: \dot{V}_{(15)}=0\right\}
$$

and $M$ be the largest invariant set within $S$. Further define

$$
\begin{aligned}
\alpha & =\sup _{\gamma \in \mathbb{R _ { + }}}\left\{\gamma: B_{\gamma G} \subset \Omega_{G} \cap \Lambda\right\}, \\
V_{\alpha}^{-1} & =\left\{(\mathbf{x}, \mathbf{y}) \in \Omega_{G}: V(\mathbf{x}, \mathbf{y}) \leqslant a(\alpha)\right\} .
\end{aligned}
$$

Then the trivial solution $(0,0)$ of the DA system is stable, $\left(\mathbf{x}(t), \mathbf{y}(t) \rightarrow M \cap V_{\alpha}^{-1}\right.$ as $t \rightarrow \infty$ and the domain of attraction contains $V_{\alpha}^{-1}$. 
Proof. Lemma 1 gives that there exists a neighbourhood $\Lambda(0)$ of the origin in which DA system (15) is equivalent to the ODE (16). Let $r \in \mathbb{R}_{+}$be such that $B_{r G} \subset \Lambda \cap \Omega_{G}$. Within $B_{r}$, the corresponding arguments for ODE systems can be used (LaSalle 1976; Rouche et al 1977).

Remarks. (a) In general, solution of (15b) yields multiple values of $\mathbf{y}$ for each $\mathbf{x}$. For each branch, system (16) and $V(\mathbf{x}, \mathbf{u}(\mathbf{x}))$ are well-defined. However, on $\Omega$, we must regard these as multi-valued. For instance, $V$ may be represented by multiple surfaces. (b) Clearly, the stability result follows easily from one for the reduced system. However, the reduced system is not usually known. So stability conditions which work directly on functions $f, g$ in (15) are needed.

\section{Stability result}

In this and the following sections, we provide some basic methedology for determining large disturbance stability of the equilibria in the DA power system model. Emphasis will be given to new insights into the nature of energy surfaces in the presence of multiple equilibria and the statement of stability results for the differential-algebraic equation model.

\subsection{Energy function (Lyapunov function candidate)}

The development of energy functions for the DA model has been studied elsewhere (Narasimhamurthi \& Musavi 1984; Hill \& Chong 1989) using first integral and Lur'e problem analysis methods. Here we summarise from Hill \& Chong (1989). A valid energy function is

$$
V\left(\omega_{g}, \mathbf{z}\right)=\frac{1}{2} \omega_{\theta}^{t} \mathbf{P}_{1}(\mu) \omega_{\theta}+\int_{z_{s}}^{z}\langle h(\lambda), \mathrm{d} \lambda\rangle
$$

where $\mathbf{z}=\left(\boldsymbol{\alpha}_{g}, \boldsymbol{\alpha}_{l},|\mathbf{V}|\right), \mathbf{h}(\mathbf{z})=\left(\tilde{\mathbf{f}}_{g}(\mathbf{z}), \mathbf{f}_{l}(\mathbf{z}), \mathbf{g}(\mathbf{z})\right)$ and $\mathbf{z}_{S}$ denotes a stable equilibrium point. $\mathbf{P}_{1}$ is given by

$$
\mathbf{P}_{1}(\mu)=q \mathbf{M}_{g}+\mu \mathbf{M}_{\boldsymbol{g}} \mathbf{l}_{m m} \mathbf{M}_{g}
$$

where $\mu$ is a scalar and $\mathbf{l}_{p q}$ denotes a $p \times q$ matrix with all its elements equal to 1 . (To simplify notation, the dimensions will be omitted.) The scalar $\mu$ is chosen to ensure $\mathbf{P}_{1}(\mu)>0$. Note that $\mathbf{P}_{1}(0)=q \mathbf{M}_{g}$. The energy function (18) can be evaluated as

$$
\begin{array}{r}
V\left(\omega_{g}, \boldsymbol{\alpha}_{g}, \boldsymbol{\alpha}_{1},|\mathbf{V}|\right)=\frac{1}{2} \omega_{g}^{t} \mathbf{P}_{1}(\mu) \omega_{g}-\frac{1}{2} \sum_{i=1}^{n} \sum_{j=1}^{n} B_{i j}\left(\left|V_{i}\right|\left|V_{j}\right| \cos \alpha_{i j}-\left|V_{i}^{S}\right|\left|V_{j}^{S}\right| \cos \alpha_{i j}^{S}\right) \\
-\int_{\alpha^{*}}^{\alpha} \tilde{\mathbf{P}^{t}} \mathrm{~d} \alpha+\sum_{i=1}^{n_{0}} \int_{\left|V_{i}^{s}\right|}^{\left|V_{i}\right|}\left[Q_{\mathrm{di}}\left(\varrho_{i}\right) / \varrho_{i}\right] \mathrm{d} \varrho_{i} .
\end{array}
$$

Define the constraint manifold

$$
G:=\left\{\mathbf{z}: \mathbf{f}_{l}(\mathbf{z})=\mathbf{0}, \mathbf{g}(\mathbf{z})=\mathbf{0}\right\} .
$$


Differentiating $V$ on $G$ gives

where

$$
\dot{V}=\frac{1}{2} \omega_{g}^{t} \mathbf{Z}_{0}(\mu) \omega_{g},
$$

$$
\mathbf{Z}_{0}(\mu)=-2 q \mathbf{D}_{g}-\mu\left(\mathbf{M}_{g} 1 \mathbf{D}_{g}+\mathbf{D}_{g} \mathbf{1} \mathbf{M}_{g}\right) .
$$

A further requirement on $\mu$ is to make $Z_{0}(\mu) \leqslant 0$. Note that $\mathbf{Z}_{0}(0) \leqslant 0$.

Clearly $\mu=0$ gives the simple kinetic energy function $\frac{1}{2} \boldsymbol{\omega}_{g}^{T} \mathbf{M}_{g} \boldsymbol{\omega}_{g}$. This remains a valid energy function for any (non-negative) values of damping $\mathbf{D}_{g}$. However, better estimates of stability regions can be obtained with a value of $\mu$ which is more closely related to the damping. This is considered in much more detail by Hill \& Chong (1989) following the results for impedance load systems by Willems (1970). In the special case of zero or uniform damping, the kinetic energy term becomes the familiar

$$
\left(1 / 2 M_{T}\right) \sum_{i=n_{0}+1}^{n-1} \sum_{j=i+1}^{n}\left(\omega_{i}-\omega_{j}\right)^{2} M_{i} M_{j} .
$$

\subsection{Stability result}

In the development of large disturbance stability results, it is useful to establish the connection between small disturbance stability and asymptotic stability of cquilibria.

Small disturbance stability refers to the stability of the linear system obtained by linearizing (9), (10). Hiskens (1990) shows that this linearization yields,

where

$$
\left[\begin{array}{c}
\Delta \dot{\alpha}_{g} \\
\Delta \dot{\omega}_{g}
\end{array}\right]=\left[\begin{array}{cc:c}
\mathbf{0} & \mathbf{T}_{g} \\
\hdashline-\mathbf{M}_{g}^{-1} \mathbf{T}_{\theta}^{j} \mathbf{F} & -\mathbf{M}_{g}^{-1} \mathbf{D}_{g}
\end{array}\right]\left[\begin{array}{c}
\Delta \boldsymbol{\alpha}_{g} \\
\Delta \omega_{g}
\end{array}\right]=\mathbf{A}\left[\begin{array}{c}
\Delta \alpha_{g} \\
\Delta \omega_{g}
\end{array}\right],
$$

$$
\mathbf{F}=\mathbf{J}_{g g}-\mathbf{J}_{g l} \mathbf{J}_{l l}^{-1} \mathbf{J}_{l g}
$$

with $\mathbf{J}_{y g}, \mathbf{J}_{g l}$ and $\mathbf{J}_{l g}$ defined in appendix $\mathbf{A}$.

It is interesting to compare the linearized system (23) with the reduced system (14). Notice that $F$ is the Jacobian of $\mathbf{P}_{g}^{*}\left(\boldsymbol{\alpha}_{\theta}\right)$. Nonsingularity of $\mathbf{J}_{l l}$ ensured the existence of functions (13), and is also required for $\mathbf{F}$ to exist.

The operating point is small disturbance stable iff all eigenvalues of $\mathbf{A}$ lie in the open left half of the complex plane. It was shown by Hiskens (1990) that the eigenvalues of $\boldsymbol{A}$ satisfied that condition if $\mathbf{F}$ was positive definite. So we can say that an equilibrium point is small disturbance stable if $\mathbf{J}_{l l}$ is nonsingular and $\mathbf{F}$ is positive definite.

Theorem 2. If an equilibrium point $\mathbf{x}_{\mathrm{e}}$ is small disturbance stable, then it is asymptotically stable in the sense of definition 2.

The proof of this is given in appendix A.

Similar results have been derived before (DeMarco \& Bergen 1984), but have relied on singular perturbation results. In so doing they have placed conditions on the sign of $\mathbf{J}_{l l}$ which are not required here.

\section{Global dynamical structure}

In this section, we move beyond the local ODE equivalence given by lemma 1 to study the DA system as globally decomposed into multiple ODE systems on regions bounded by surfaces of algebraic singularity. 
The assumption of constant real power load made at (2a) was necessary to ensure path independence of the energy function integral in (18). However, such an assumption is not required when considering structural aspects of DA systems. It shall be relaxed for this discussion, i.e., we will allow real power loads to take the form $P_{d i}=P_{d i}^{0}\left(\left|V_{i}\right|\right)$.

It can be seen from (8) and (12) that real power loads influence $\boldsymbol{J}_{l l}$. When real power loads are constant $J_{l l}$ is symmetric, so its eigenvalues are all real. However voltage dependence of real power loads causes $\mathbf{J}_{l l}$ to be asymmetric. This can (and often does) cause $\mathbf{J}_{n}$ to have complex eigenvalues.

\subsection{ODE decomposition}

Our first result will establish the ODE decomposition.

It is convenient to make the following assumption.

A4. For all $\mathbf{z} \in G$, all negative real eigenvalues of $\left.\mathbf{J}_{\| l}\right|_{\mathbf{z}}$ are distinct.

This assumption eliminates the possibility that as a path over the constraint manifold is traversed, negative real eigenvalues of $\mathbf{J}_{\|}$could merge, then split from the real axis as a complex pair. Under this assumption, the only way that the number of negative real eigenvalues of $\mathbf{J}_{11}$ can change is by a real eigenvalue crossing the imaginary axis, i.e., $\mathbf{J}_{n l}$ going singular. The assumption is valid for most realistic power systems. However rare circumstances can be found where it is not true (Hiskens 1990). The consequences of such behaviour are explored later.

Open sets $C_{l}$ which lie within the constraint manifold can now be defined as

$$
C_{l}=\left\{\mathbf{z} \in G:\left.\operatorname{det} \mathbf{J}_{l l}\right|_{\mathbf{z}} \neq 0,\left.\mathbf{J}_{l l}\right|_{\mathbf{z}} \text { has } l \text { negative real eigenvalues }\right\} \text {. }
$$

These sets may not necessarily be connected. Partition each $C_{l}$ into its connected components $C_{l 1}, \ldots C_{l k}$, i.e.

$$
C_{l}=\bigcup_{i=1}^{k} C_{l i} \text { and } C_{l i} \cap C_{l j}=\phi, i \neq j, i, j=1 . . k \text {. }
$$

From (13), it can be seen that the functions $\phi, \psi$, which enable the establishment of the equivalent $O D E$ representations, are functions of generator angles only. Therefore it is convenient to project the sets $C_{l i}$ onto their generator angle components, as

$$
A_{l i}=\left\{\alpha_{g}:\left(\alpha_{g}, \alpha_{l},|\mathbf{V}|\right) \in C_{l i}\right\}
$$

A5. Each $C_{l i}$ is simply connected.

This assumption is difficult to check. However, in the context of power systems, extensive studies have not revealed a counter-example.

Theorem 3. Assume each $C_{l i}$ is simply connected. On each $C_{l i}$, the set $G$ is represented by unique continuous functions $\boldsymbol{\phi}_{1 i}: A_{l i} \rightarrow \mathbb{R}^{n_{0}}, \boldsymbol{\psi}_{l i}: A_{l i} \rightarrow \mathbb{R}^{n_{0}}$ such that $\boldsymbol{\alpha}_{l}=\phi_{l i}\left(\boldsymbol{\alpha}_{g}\right)$, $|\mathbf{V}|=\psi_{l i}\left(\boldsymbol{\alpha}_{y}\right)$. The DA system (9), (10) is equivalent to the local ODE representation (14).

The proof is given in appendix $B$.

Remarks. (a) It is easy to generate examples of systems where the sets $C_{l}$ are not connected (Hiskens 1990). 
(b) The sizes of sets $C_{l i}$ depend greatly on the load model parameters. Conditions can be given for ensuring $C_{l}, l \neq 0$ are empty (Hiskens \& Hill 1989; Hiskens 1990).

(c) This result sharpens the one given earlier by Hiskens \& Hill (1989).

The boundaries of the sets $C_{l i}$ are referred to as "impasse surfaces" - a term borrowed from circuit theory (Hasler \& Neirynck 1986).

The regions $C_{l i}$ are referred to as voltage causal regions. Within any $C_{l i}$, the load bus voltages and angles are continuously dependent on the generator angles. If trajectories meet an impasse surface, voltage behaviour can no longer be predicted from the DA model.

The impasse surface $I$ is given by

Note that

$$
I=\left\{\mathbf{z} \in G:\left.\operatorname{det} \mathbf{J}_{\| l}\right|_{\mathbf{z}}=0\right\} \text {. }
$$

$$
G=\left(\bigcup_{i=0}^{2 n_{0}} C_{i}\right) \cup l .
$$

Define augmented algebraic constraint function

$$
\mathrm{i}(\mathrm{z})=\left(\operatorname{det} \mathrm{J}_{l l}, \mathrm{f}_{l}, \mathrm{~g}\right)
$$

Fact. Suppose rank $D \mathrm{i}=2 n_{0}+1$ at a point $p$ in $I$. Then in a neighbourhood of $p$, $I$ is a differentiable $(m-2)$-manifold.

From this fact we can build a picture of $I$ as composed of intersecting differentiable $(m-2)$-manifolds. On each of these manifolds, $\mathbf{J}_{l l}$ has exactly one zero eigenvalue. They intersect at lower dimensional manifolds where 2 or more eigenvalues are zero and rank $D i<2 n_{0}+1$. It remains to determine whether $D i$ has full rank at all non-intersection points, i.e. does some $(m-2)$-manifold segment have a boundary?

The following example examines the constraint manifold structure in the context of power systems.

Example 1. Consider the network shown in figure 1. For simplicity we will not augment this network by buses representing fictitious generator internal buses. The dynamic variables are therefore $\alpha_{g 1}, \alpha_{g 2}, \omega_{g 1}, \omega_{g 2}, \omega_{g 3}$. The algebraic variables are

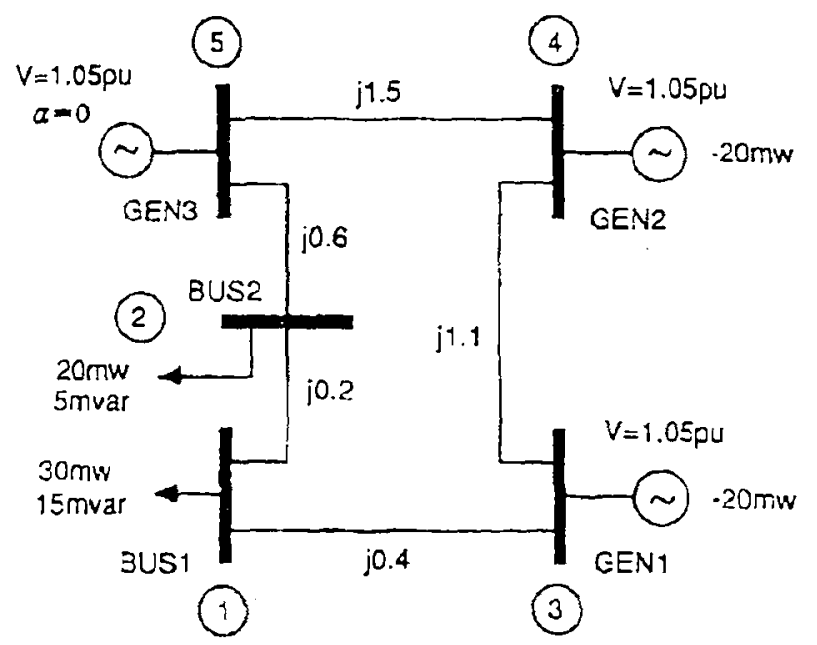

Figure 1. Power system network. 


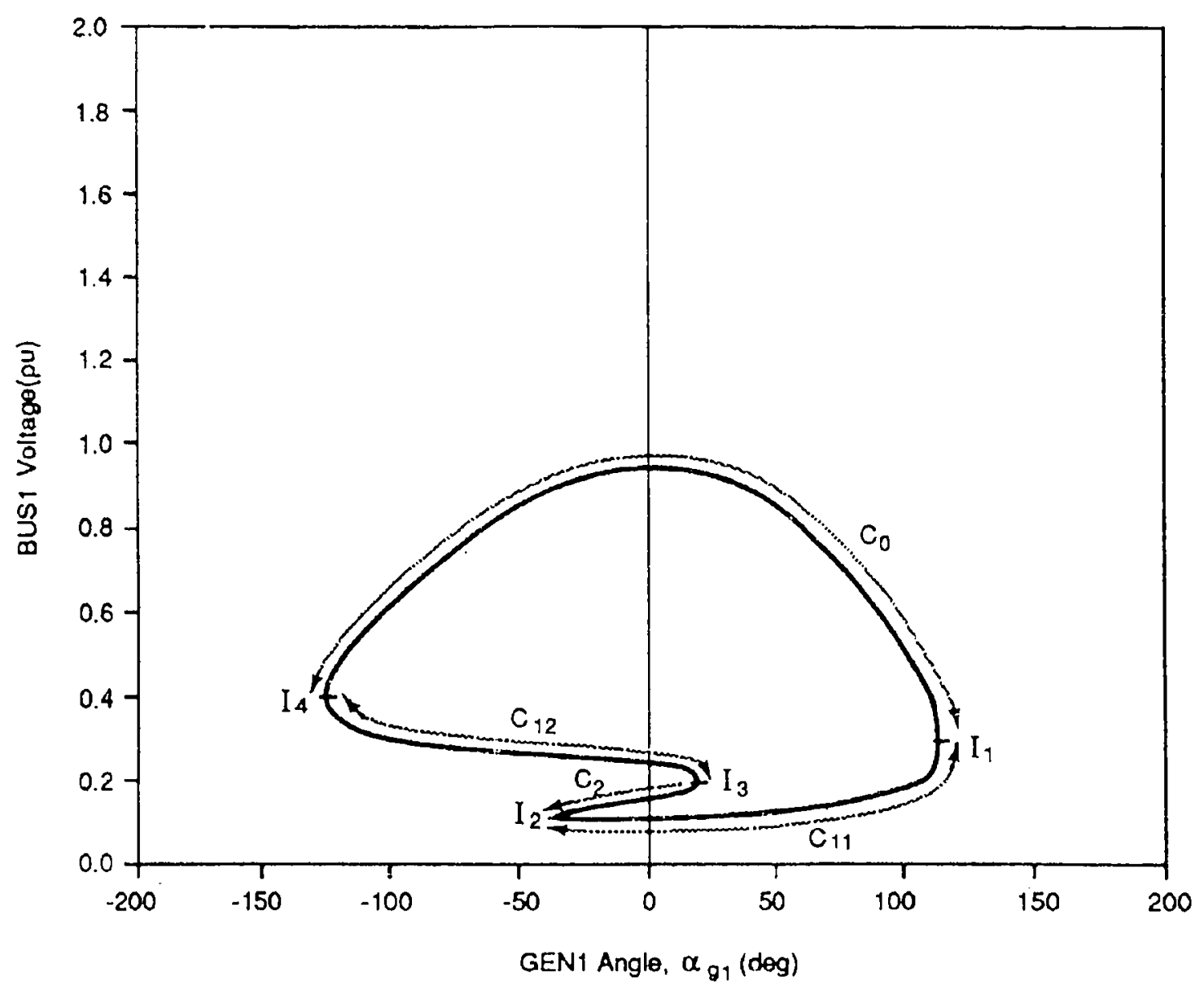

Figure 2. Constraint manifold projection, $\left(x_{g 1},\left|V_{1}\right|\right.$ space $)$.

$\alpha_{11}, \alpha_{l 2},\left|V_{1}\right|,\left|V_{2}\right|$. The constraint mainfold has dimension 2. Note though that because the load bus network is connected between generator GEN1 and the reference bus GEN3, the load bus variables depend only on $\alpha_{\theta 1}$. They are independent of $\alpha_{g 2}$. Therefore the constraint manifold has a cylindrical form in the sense that the same shape is maintained at all values of $\alpha_{\theta 2}$. Projections of a slice through this 2-manifold are shown in figure 2. The impasse surface appears in these projections as four points $I_{1}, I_{2}, I_{3}, I_{4}$, separating causal regions $C_{0}, C_{11}, C_{32}, C_{2}$. The impasse surface segments do not intersect.

The impasse surface, which divides the constraint manifold into causal regions, is defined at (27) as the set of points where $\mathbf{J}_{l}$ is singular. It is therefore composed of points at which at least one real eigenvalue of $\mathbf{J}_{\|}$is zero. Note then that complex eigenvalues have no influence on the structure of the impasse surface. Hence, the causal region decomposition is not influenced at all by complex eigenvalue behaviour. (Because of its non-zero imaginary part, a complex eigenvalue can have zero real part at a causal point.)

However, it can be observed that along a path traversing the constraint manifold, the imaginary part of a complex pair may become zero, leaving a pair of repeated real eigenvalues. These real eigenvalues can then of course influence the decomposition in the usual way. Whilst not affecting the causal region structure, this behaviour does cause difficulties with the indexing scheme established for the regions in (25). As an 
example, if the imaginary part of a complex pair, which was in the left half plane, became zero, two negative real eigenvalues would be created. Points in the same causal region would have causal indices differing by two. However, because the indexing scheme is no more than a convenient way of identifying regions, this effect is not considered important. Certainly such eigenvalue behaviour in no way affects the analysis of DA systems.

Thus the global structure of DA is established: the constraint set $G$ consists of disjoint open sets $C_{1 i}$ which are separated by the impasse surface and within which the dynamics are given by a local ODE description.

\subsection{Conditions for global voltage causality}

The above presents a complicated general picture for the dynamical structure of DA systems. For the power systems case, variations of the load indices cause significant structural changes to the causal region/impasse surface decomposition of the constraint manifold. It is possible to find load indices which ensure global voltage causality, i.e., det $\mathbf{J}_{u l} \neq 0$ at all points on the constraint manifold. Then the DA model which employed those indices could be globally reduced to a unique set of differential equations, valid at all points on the constraint manifold. Results for special cases are available (Hiskens \& Hill 1989; Hiskens 1990).

\section{Stability assessment}

In this section the implications of the global structure on the energy function picture are studied.

\subsection{Estimate of stability region}

In theorem 3, local solvability of (10) was extended to solvability over voltage causal regions. The same concept can be used to extend the region of validity of the local representation of $V$. An estimate for the region of attraction for a stable equilibrium point, $\mathbf{z}_{S}$, of the DA model can then be determined. Let the number of negative eigenvalues of $\left.J_{n l}\right|_{z_{s}}$ be $l$. By theorem 3 there exist unique continuous functions $\boldsymbol{\alpha}_{l}=\phi_{l i}\left(\boldsymbol{\alpha}_{g}\right),|\mathrm{V}|=\psi_{l i}\left(\boldsymbol{\alpha}_{\theta}\right)$ such that over a voltage causal region $C_{l i}, V$ at $(18)$ can be written

$$
\begin{aligned}
V_{l i}\left(\omega_{g}, \boldsymbol{\alpha}_{g}\right) & =\left.V\left(\omega_{g}, \mathbf{z}\right)\right|_{C_{l l}} \\
& =\frac{1}{2} \omega_{g}^{t} \mathbf{P}_{1}(\mu) \omega_{g}+\int_{\alpha_{g}^{s}}^{\alpha_{g}}\left\langle\tilde{\mathbf{f}}_{g}\left(\boldsymbol{\alpha}_{g}, \boldsymbol{\phi}_{l i}\left(\boldsymbol{\alpha}_{g}\right), \psi_{l i}\left(\boldsymbol{\alpha}_{g}\right)\right), \mathrm{d} \boldsymbol{\alpha}_{g}\right\rangle .
\end{aligned}
$$

Define the sets

$$
\begin{aligned}
& R_{l i}^{k}=\left\{\left(\omega_{g}, \boldsymbol{\alpha}_{g}\right) \mid V_{l i}\left(\omega_{g}, \boldsymbol{\alpha}_{g}\right) \leqslant k\right\}, \\
& S_{l i}^{k}=\left\{\left(\boldsymbol{\alpha}_{g}, \boldsymbol{\alpha}_{l},|\mathbf{V}|\right):\left(\omega_{g}, \boldsymbol{\alpha}_{g}\right) \in R_{l i}^{k}, \boldsymbol{\alpha}_{l}=\phi_{l i}\left(\boldsymbol{\alpha}_{g}\right),|\mathbf{V}|=\psi_{l i}\left(\boldsymbol{\alpha}_{g}\right)\right\} .
\end{aligned}
$$

Note that the elements of $S_{l i}^{k}$ are simply points in $\left(\boldsymbol{\alpha}_{g}, \omega_{l},|\mathbf{V}|\right)$-space which correspond to elements of $R_{l i}^{k}$ (i.e., points in $\left(\omega_{g}, \boldsymbol{\alpha}_{g}\right)$-space).

An estimate of the stability region is obtained via the following theorem. 
Theorem 4. Let $\xi_{S}=\left(\mathbf{0}, \mathbf{x}_{S}\right)$ be an asymptotically stable equilibrium point of the $D A$ model. Then, for all $k>0$ such that $R_{l i}^{k}$ is bounded and $S_{l i}^{k} \subseteq C_{l i}$ any trajectory $s\left(t, \mathbf{x}_{0}\right)$ with initial conditions $\mathbf{x}_{0} \in R_{l i}^{k}$ has the following properties:

(i) $\mathbf{s}\left(t, \mathbf{x}_{0}\right) \in R_{l i}^{k}$, for all $t \geqslant 0$ (i.e. $R_{l i}^{k}$ is invariant with respect to the $D A$ model).

(ii) $\mathbf{s}\left(t, \mathbf{x}_{0}\right) \rightarrow \mathbf{x}_{s}$ as $t \rightarrow \infty$.

The proof of this theorem is given in appendix $\mathrm{A}$.

Remarks. (a) As $k$ is increased, a value will be attained where one of the two conditions on $R_{l i}^{k}, S_{l i}^{k}$ breaks down. Either

(i) $R_{l i}^{k}$ becomes unbounded, i.e. $V_{l i}$ is no longer locally positive definite; or

(ii) $S_{l i}^{k} \nsubseteq C_{l i}$, i.e. there are points in $S_{l i}^{k}$ for which the local model is no longer valid.

These phenomena are consistent with definitions of power system stability (Hiskens $\&$ Hill 1989). The limit placed on $k$ by (i) ensures that all points in $R_{l i}^{k}$ are attracted to the stable equilibrium point, $\mathbf{z}_{s}$, i.e., if $k$ was allowed to increase, then for some $\mathbf{x}_{0} \in R_{l i}^{k}, \mathbf{s}\left(t, \mathbf{x}_{0}\right) \nrightarrow \mathbf{x}_{s}$, as $t \rightarrow \infty$. This is angle instability because the dynamic variables, i.e. generator frequencies and angles, do not tend towards the stable equilibrium point. (Note though that the impasse surface could be encountered as the system proceeded along this unstable trajectory. In that case voltage causality would be lost as a consequence of the initial angle instability.) The limit placed by (ii) ensures that the local model and energy function are valid for all points in $S_{l i}^{k}$. In this case, if $k$ was to increase, then for some $\mathbf{z}_{0} \in S_{l l}^{k}$, det $\mathbf{J}_{u}=0$, i.e., lack of voltage causality.

(b) Let the largest value of $k$ satisfying theorem 4 be $k_{\text {crit }}$. This value could be used in the traditional way as the critical value of energy able to be attained by the disturbed system with stability still guaranteed. This of course is likely to be quite conservative. A practical algorithm will employ information on fault location (Pai 1981).

(c) A result similar to this, but requiring all eigenvalues of $\mathbf{J}_{l l}$ to be positive has been derived by DeMarco \& Bergen (1984). Singular perturbation results were used in that case.

\subsection{Multiple energy function sheets}

If the energy function (18) is treated in the usual way as the sum of kinetic and potential energy terms, then it is only the potential energy term which is dependent on the set $C_{l i}$. The local potential energy functions are functions of $\boldsymbol{\alpha}_{\theta}$ only, and so can be conceptualized as ( $m-1$ )-hypersurfaces (or sheets) in $\boldsymbol{\alpha}_{g}$-space. (Recall the potential energy well concept in energy function methods, Pai 1981).

For each region $C_{i i}$ defined by theorem 3, a unique local potential energy function exists, each one a sheet in $\alpha_{g}$-space. It is not difficult to imagine therefore how it is possible to have a number of asymptotically stable equilibria. (Those sheets with a locally positive definite section must have an asymptotically stable equilibrium point at the lowest point of that section.) Note that not all sheets need contain equilibria however.

All the PE sheets join on the impasse surface. The sheets can be thought of as approaching each other infinitesimally closely at the impasse surface.

While not hard to illustrate by example (Hiskens \& Hill 1989), a complete theoretical 
discussion of these issues remains to be studied. Nevertheless it is already clear that DA models change the traditional view of large disturbance stability substantially. For instance, the phenomenon of short-term voltage collapse can perhaps be explained in terms of "jumps" between different energy levels (Hiskens \& Hill 1989).

\section{Appendix A. Proofs of stability results}

Proof of theorem 2

Define the Jacobian

$$
\mathbf{J}=\partial \mathbf{h} / \partial \mathbf{z}=\left[\begin{array}{c:cc}
\partial \tilde{\mathbf{f}}_{g} / \partial \boldsymbol{\alpha}_{g} & \partial \tilde{\mathbf{f}}_{g} / \partial \boldsymbol{\alpha}_{l} & \partial \tilde{\mathbf{f}}_{g} / \partial|\mathbf{V}| \\
\hdashline \partial \mathbf{f}_{l} / \partial \boldsymbol{\alpha}_{g} & & \mathbf{J}_{l l} \\
\partial \mathbf{g} / \partial \boldsymbol{\alpha}_{g} &
\end{array}\right]=\left[\begin{array}{ll}
\mathbf{J}_{g g} & \mathbf{J}_{g l} \\
\mathbf{J}_{l g} & \mathbf{J}_{l l}
\end{array}\right],
$$

where $h$ is defined after $(18)$. Let $\xi=\left(\omega_{g}, \mathbf{z}\right)=(\mathbf{x}, \mathbf{y})$, where $\mathbf{x}=\left(\omega_{g}, \boldsymbol{\alpha}_{g}\right)$ contains the dynamic states and $\mathbf{y}=\left(\boldsymbol{\alpha}_{1},|\mathbf{V}|\right)$ contains the algebraic states.

If $\boldsymbol{z}_{e}$ is small disturbance stable, then $\left.\operatorname{det} \mathbf{J}_{l l}\right|_{\mathbf{x}_{e}} \neq 0$ and $\left.\mathbf{F}\right|_{\boldsymbol{r}_{e}}=\mathbf{J}_{g g}-\left.\mathbf{J}_{g l} \mathbf{J}_{l l}^{-1} \mathbf{J}_{l_{g}}\right|_{\mathbf{z}_{e}}$ is positive definite.

Observe that $\tilde{\mathbf{f}}_{\boldsymbol{\theta}}\left(\boldsymbol{z}_{\boldsymbol{e}}\right)=\mathbf{0}$. Hence $\left.(\partial V / \partial \xi)\right|_{\xi_{e}}=0$, and a Taylor expansion of $V(\xi)$ about $\xi_{e}$ yields,

$$
V(\xi)=\left(\xi-\xi_{e}\right)^{r}\left[\begin{array}{cc}
\mathbf{P}_{1}(\mu) & 0 \\
0 & \mathbf{J}_{z_{e}}
\end{array}\right]\left(\xi-\xi_{e}\right)+0\left(\left\|\xi-\xi_{e}\right\|^{3}\right),
$$

where $0(\cdot)$ represents higher order terms.

Now note that

So

$$
\left[\begin{array}{l}
\mathrm{d} \mathbf{f}_{l} \\
\mathrm{~d} \mathbf{g}
\end{array}\right]=\mathbf{J}_{l g} \mathrm{~d} \boldsymbol{\alpha}_{g}+\mathbf{J}_{l l}\left[\begin{array}{l}
\mathrm{d} \boldsymbol{\alpha}_{1} \\
\mathrm{~d}|\mathbf{V}|
\end{array}\right]=\mathbf{0}
$$

$$
\left[\begin{array}{l}
\mathrm{d} \boldsymbol{\alpha}_{1} \\
\mathrm{~d}|\mathbf{V}|
\end{array}\right]=\mathbf{J}_{l i}^{-1} \mathbf{J}_{l g} \mathrm{~d} \boldsymbol{\alpha}_{g}
$$

By hypothesis $\left.\mathbf{J}_{l l}\right|_{\mathbf{r}}$ is nonsingular, so

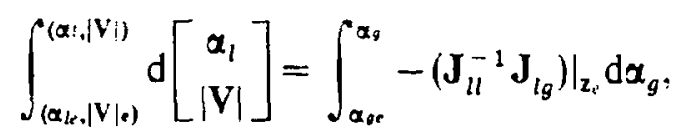

$$
\begin{aligned}
& {\left[\begin{array}{c}
\alpha_{l} \\
|\mathbf{V}|
\end{array}\right]-\left[\begin{array}{c}
\alpha_{l e} \\
|\mathbf{V}|_{e}
\end{array}\right]=-\left.\left(J_{l l}^{-1} \mathbf{J}_{l g}\right)\right|_{z_{e}}\left(\alpha_{g}-\alpha_{g e}\right) .}
\end{aligned}
$$

From (A1), $V(\xi)$ can be rewritten

$$
V(\xi)=\left[\begin{array}{c}
\omega_{g}-\omega_{g e} \\
\alpha_{g}-\alpha_{g e} \\
\alpha_{l}-\alpha_{l e} \\
|\mathbf{V}|-|\mathbf{V}|_{e}
\end{array}\right]^{\prime}\left[\begin{array}{ccc}
\mathbf{P}_{1}(\mu) & 0 & 0 \\
0 & \mathbf{J}_{g g} & \mathbf{J}_{g l} \\
0 & \mathbf{J}_{l g} & \mathbf{J}_{l l}
\end{array}\right]\left[\begin{array}{c}
\omega_{g}-\omega_{g e} \\
\alpha_{g}-\alpha_{g e} \\
\alpha_{l}-\alpha_{l e} \\
|\mathbf{V}|-|\mathbf{V}|_{e}
\end{array}\right]+0(\cdot),
$$


where all partial derivatives are evaluated at $\mathbf{z}_{e}$. Combining (A2) and (A3) gives

$$
\begin{array}{r}
V(\alpha)=\left(\omega_{g}-\omega_{g e}\right)^{t} \mathbf{P}_{1}(\mu)\left(\omega_{g}-\omega_{g e}\right)+\left.\left(\alpha_{g}-\alpha_{g e}\right)^{t}\left(\mathbf{J}_{g g}-\mathbf{J}_{g} \mathbf{J}_{l l}^{-1} \mathbf{J}_{l g}\right)\right|_{\mathbf{z}_{e}}\left(\alpha_{g}-\alpha_{g e}\right) \\
+0(\cdot)
\end{array}
$$

It is given that $\mathbf{P}_{\mathrm{I}}(\mu)>0$. Also, the assumption of small disturbance stability implies

$$
\left.\left(J_{g g}-\mathbf{J}_{g l} \mathbf{J}_{l l}^{-1} \mathbf{J}_{l g}\right)\right|_{z_{\bullet}}>0 \text {. }
$$

Thus $V\left(\xi_{e}+\zeta\right)$ is locally positive definite on some neighbourhood of $\xi_{e}$.

Define

$$
R_{k}=\text { component of }\{\xi: V(\xi) \leqslant k\} \text { containing } \xi_{e} .
$$

Because det $\mathbf{J}_{n} \neq 0$ by hypothesis, there exists a neighbourhood of $\xi_{e}$ such that the algebraic equations (10) are solyable. Thus in the whole state space, there is a neighbourhood $\Lambda\left(\mathbf{x}_{e}\right)$ as defined for lemma 1 .

Now consider

$$
S=\left\{\xi: \dot{V}_{\mathrm{DA}}=0\right\}
$$

where $\dot{V}_{D A}$ is the derivative of $V$ along trajectories of the DA model (9), (10). From (21),

$$
\dot{V}_{\mathrm{DA}}=\frac{1}{2} \omega_{g}^{t} \mathrm{Z}_{0}(\mu) \omega_{\mathrm{B}},
$$

with $\mathrm{Z}_{0}(\mu)$ given by (22). Therefore, it is easy to see that $S$ consists of all equilibria $\left(\mathbf{0}, \mathbf{z}_{\mathfrak{e}}\right)$ where $\mathbf{z}_{e}$ satisfies (11).

Because $V$ is locally positive definite, it is clear that for some $k$, sufficiently small $R_{k}$, is bounded and $S \cap R_{k_{1}}=\left\{\xi_{e}\right\}$.

The result then follows from theorem 1 .

Proof of theorem 4. Because $S_{l i}^{k} \subseteq C_{l i}$ the local energy function $V_{l i}\left(\omega_{g}, \alpha_{g}\right)$ is valid for all $\left(\omega_{g}, \alpha_{g}\right) \in R_{l i}^{k}$. As in the proof of theorem 2, differentiating $V_{l i}$ along trajectories of the reduced ODE system (14) gives

$$
\dot{V}_{l i}\left(\omega_{g}, \alpha_{\theta}\right)=\omega_{g}^{z} Z_{0}(\mu) \omega_{\theta}
$$

i.e., $\dot{V}_{l i}\left(\omega_{g}, \alpha_{g}\right) \leqslant 0$.

Due to the construction of $R_{l i}^{k}$ and because $\dot{V}_{l l}\left(\omega_{g}, \alpha_{g}\right) \leqslant 0$, all trajectories $\xi\left(t, \xi_{0}\right)$ of the DA model with initial conditions $\mathbf{x}_{0} \in R_{l i}^{k}, \mathbf{z}_{0} \in S_{l i}^{k}$ must be such that $\mathbf{x}\left(t, \xi_{0}\right)$ remains in $R_{l i}^{k}$ (property i).

Property ii follows from theorem 1 and property $i$.

\section{Appendix B. Proof of theorem 3.}

Because $C_{l i}$ is open, each point in $C_{t i}$ has a neighbourhood contained in $C_{l i}$. Further, as $C_{l i}$ is simply connected, it is pathwise connected, i.e., between any two points in $C_{l i}$ there exists a path which is completely contained in $C_{l i}$.

The definition of $C_{l i}$ implies that the implicit function theorem is valid at all points $\left(\boldsymbol{\alpha}_{g}^{\prime}, \boldsymbol{\alpha}_{1}^{\prime},|\mathbf{V}|^{\prime}\right) \in C_{l i}$. Therefore, within a neighbourhood of any point $\left(\boldsymbol{\alpha}_{g}^{\prime}, \boldsymbol{\alpha}_{1}^{\prime},|\mathbf{V}|^{\prime}\right)$ there 
exist unique continuous functions $\phi_{l i}^{\prime}, \psi_{l i}^{\prime}$ which relate $\alpha_{l},|\mathbf{V}|$ to $\alpha_{g}$. (Continuity follows from the fact that $\mathbf{f}_{l}, \mathbf{g}$ are continuous.)

Consider two arbitrary points $\mathbf{z}_{1}, \mathbf{z}_{2} \in C_{l i}$ sufficiently close that neighbourhoods of these points, $U_{1}, U_{2} \subset C_{l i}$, overlap, i.e. $U_{1} \cap U_{2} \neq \phi$. There exist on $U_{1}$ unique continuous functions $\phi_{l i}^{1}, \psi_{l i}^{1}$, whilst on $U_{2}$ there exist unique continuous functions $\boldsymbol{\phi}_{l i}^{2}, \boldsymbol{\psi}_{l i}^{2}$. Now consider a point $z_{3}=\left(\alpha_{g}^{3}, \alpha_{l}^{3},|\mathbf{V}|^{3}\right) \in U_{1} \cap U_{2}$. Because this point belongs to both neighbourhoods, it must satisfy $\phi_{i i}^{1}, \psi_{i i}^{1}$ and $\phi_{i i}^{2}, \psi_{i i}^{2}$, i.e.

and

$$
\alpha_{1}^{3}=\phi_{l i}^{1}\left(\alpha_{g}^{3}\right) \text { and }|\mathbf{V}|^{3}=\psi_{l i}^{1}\left(\alpha_{g}^{3}\right)
$$

$$
\alpha_{l}^{3}=\phi_{l i}^{2}\left(\alpha_{g}^{3}\right) \text { and }|V|^{3}=\psi_{l i}^{2}\left(\alpha_{g}^{3}\right),
$$

But $\phi_{l i}^{1}, \psi_{l i}^{1}$ and $\phi_{l i}^{2}, \psi_{l i}^{2}$ are unique at all points within their respective neighbourhoods. So;

$$
\phi_{l i}^{1}=\phi_{1 i}^{2} \text {, and } \psi_{1 i}^{1}=\psi_{1 i}^{2} .
$$

Because points $\mathbf{z}_{1}, \mathbf{z}_{2}$ are arbitrary, the above argument applies for any sufficiently close points. In particular, along any path completely contained in $C_{1 i}$ every point has a neighbourhood which intersects neighbourhoods of other points on the path. A chain of points with overlapping neighbourhoods can be formed along the path. By applying the above argument at successive points along that chain and since $C_{l i}$ is simply connected, it can be concluded that functions $\phi_{1 i}$ and $\psi_{1 i}$ must be unique along the whole path, including its end points.

But any two points in $C_{l i}$ can be joined by some path. Further, because $C_{l i}$ is simply connected, paths can be continuously transformed within $C_{l i}$. Therefore $\phi_{l i}$ and $\psi_{l i}$ are unique over $C_{l i}$.

\section{References}

Bergen A R, Hill D J 1981 A structure preserving model for power system stability analysis. IEEE Trans. Power Appar. Syst. PAS-100: 25-35

Chiang H-D, Fekih-Ahmed L 1992 On the direct method for transient stability analysis of power system structure preserving models. IEEE International Symposium on Circuits and Systems (New York: IEEE)

DeMarco C L, Bergen A R 1984 Application of singular perturbation techniques to power system transient stability analysis. Memo M. 84/7, Electronics Research Laboratory, University of California, Berkeley

Fleming W 1977 Functions of several variables (New York: Springer-Verlag)

Hasler M, Neirynck J 1986 Nonlinear circuits (Norwood, MA: Artech House)

Hill D J, Chong CN 1989 Lyapunov functions of Lur'e-Postnikov form for structure preserving models of power systems. Automatica 25: 453-460

Hill D J, Mareels I A 1990 Stability theory for differential/algebraic systems with application to power systems. IEEE Trans. Circuits Syst. CAS-37: 1416-1423

Hiskens I A 1990 Energy functions, transient stability and voltage behaviour in power systems, $\mathrm{Ph} \mathrm{D}$ thesis, University of Newcastle

Hiskens I A, Hill D J 1989 Energy functions, transient stability and voltage behaviour in power systems with nonlinear loads. IEEE Trans. Power Syst. 4: 1525-1533

Kwatny H G, Pasrija A K, Bahar L Y 1986 Static bifurcations in electric power networks: loss of steady-state stability and voltage collapse. IEEE Trans. Circuits Syst. CAS-33: $981-991$

LaSalle J P 1976 The stability of dynamical systems (Philadelphia: SIAM)

Narasimhamurthi N, Musavi M T 1984 A generalized energy function for transient stability analysis of power systems. IEEE Trans. Circuits Syst. CAS-31: 637-645 
Pai M A 1981 Power system stability (New York: North-Holland)

Rouche N, Habets P, Laloy M 1977 Stability theory by Lyapunov's direct method. Applied Mathematical Sciences, Vol. 22 (New York: Springer-Verlag)

Venkatasubramanian V, Schattler H, Zaborszky J 1991 A taxonomy of the dynamics of the large power system with emphasis on its voltage stability. Proc. Bulk Power System Voltage Phenomena, Voltage Stability and Security, ECC/NSI Workshop, Deep Creek Lake, MD

Willems J L 1970 Optimum Lyapunov functions and stability regions for multimachine power systems. Proc. Inst. Elec. Eng. 117: 573-577 